\title{
Strategies to Increase the Participation Rate of Colorectal Cancer Screening
}

\author{
Yoon Suk Jung \\ Division of Gastroenterology, Department of Internal Medicine, Kangbuk Samsung Hospital, Sungkyunkwan University School of Medicine, \\ Seoul, Korea
}

See "Increase in the Colorectal Cancer Screening Rate by a Round-Mailed Fecal Immunochemical Testing Kit and Associated Factors in Underserved Regions of Korea: A Community-Based Intervention Study" by Seri Hong, et al. on page 323, Vol. 14, No. 3, 2020

Colorectal cancer (CRC) is one of the most common malignancies and remains the leading cause of cancer-related mortality worldwide. ${ }^{1,2}$ To reduce the incidence and mortality of CRC, it is important to increase the participation rate of the CRC screening program. ${ }^{3}$ Fecal immunochemical test (FIT) is currently regarded as the best available noninvasive CRC screening tool. The U.S. Multi-Society Task Force on CRC recommends FIT as the first-tier screening method for CRC, along with colonoscopy. ${ }^{4}$ The U.S. Multi-Society Task Force on CRC recommends FIT completion rate to those offered testing of $60 \%$ or greater. $^{5}$ Several countries, including South Korea, have adopted FIT in nationwide population-based CRC screening programs. In the Korean National Cancer Screening Program (NCSP), the participation rate has been reported to be lower for CRC screening than other cancers, such as stomach, breast, and cervix cancer; the participation rate was 26\%-33\% from 2009 to $2012 .^{6}$

In this issue of Gut and Liver, Hong et al. ${ }^{7}$ conducted a community-based intervention study to evaluate the impact of the round-mailed FIT kit on the CRC screening rate in underserved regions, and to identify factors related to nonparticipation. Residents were recruited from three rural regions (Jangsu, Goseong, and Uiryeong) lacking NCSP screening units, between 2015 and 2016. A package containing a FIT kit and a return envelope addressed to the local health center was distributed to each subject by mail. Thirty days after postage of the FIT kit, nonresponders were reminded via telephone as the second intervention. They reported that CRC screening participation rates increased from $24.5 \%$ to $42.6 \%$ as a result of postal screening and increased further to $51.4 \%$ after the telephone reminder. ${ }^{7}$ The interven- tion of delivering FIT kits to residents of rural areas, followed by phone call reminders for nonresponders, resulted in an increase of about 2.1-fold in the CRC screening participation rate. They also showed that factors associated with nonparticipation were low education level and poor previous participation in the NCSP. ${ }^{7}$

Although this study is the first intervention study to assess the effectiveness of the round-mailed FIT kit in Korea, many studies on this topic have already been conducted in other countries. It is an expected result, of course, but previous studies have also shown that mailed outreach is superior to usual care alone for CRC screening based on fecal tests. ${ }^{8,9}$ Recently, a meta-analysis study of seven randomized controlled trials including 12,501 subject ( $\mathrm{n}=5,703$ assigned mailed outreach and $\mathrm{n}=6,798$ usual care) in the United States suggests that mailed outreach offering a guaiac fecal occult blood test (gFOBT) or FIT is a highly effective, evidence-based strategy for increasing CRC screening, including among underserved populations. More specifically, this meta-analysis study demonstrated that mailed outreach resulted in a $28 \%$ absolute and a 2.8 -fold relative increase in screening completion compared to usual care. ${ }^{8}$ Similarly, another comprehensive systematic review of randomized trials and observational studies revealed that multicomponent interventions such as mailing FIT/FOBT materials directly to patient's homes, use of pre-addressed stamped envelopes to facilitate kit return, and client reminder and recall were highly effective strategies for improving gFOBT/FIT completion rate in rural and low-income populations in the United States. ${ }^{9}$

Similar to the study by Hong et al., a previous study evalu-

Correspondence to: Yoon Suk Jung

Division of Gastroenterology, Department of Internal Medicine, Kangbuk Samsung Hospital, Sungkyunkwan University School of Medicine, 29 Saemunan-ro, Jongno-gu, Seoul 03181, Korea

Tel: +82-2-2001-8577, Fax: +82-2-2001-2049, E-mail: ys810.jung@samsung.com pISSN 1976-2283 eISSN 2005-1212 https://doi.org/10.5009/gnl20110

(c) This is an Open Access article distributed under the terms of the Creative Commons Attribution Non-Commercial License (http://creativecommons.org/licenses/by-nc/4.0) which permits unrestricted non-commercial use, distribution, and reproduction in any medium, provided the original work is properly cited. 
ated the effectiveness of interventions to increase CRC screening in Korea. ${ }^{10}$ This Korean study compared the participation rates in CRC screening among four groups: control, postal intervention, telephone intervention, and telephone and postal intervention. ${ }^{10}$ This study showed that subjects who received telephone and postal intervention were most likely $(27.8 \%)$ to participate in CRC screening, followed by subjects who received telephone intervention only (24.3\%), postal intervention only (16.5\%), and subjects in the control group (13.5\%). ${ }^{10}$ Combined telephone and postal intervention and telephone only intervention resulted in increased rates of participation in CRC screening compared to the control group, while there was no significant difference between the postal intervention only and control groups. These results suggest that postal intervention alone may not be sufficient to increase the rate of CRC screening.

Given these results, it may be more effective to perform both mail and phone calls simultaneously as the first intervention rather than phone call reminders for nonresponders as the second intervention. In other words, the optimal strategy to increase FIT completion rate may be by telephone to instruct the screenees to collect stool using the FIT kit and return the stool sample using the enclosed envelope. These instructions would be most effective if provided when sending the FIT kit. These aggressive interventions will be especially necessary for persons who are less likely to participate in CRC screening, such as persons with low education level, those who have poorly participated in previous screening, or rural residents.

\section{CONFLICTS OF INTEREST}

No potential conflict of interest relevant to this article was reported.

\section{ORCID}

Yoon Suk Jung

https://orcid.org/0000-0002-1963-7170

\section{REFERENCES}

1. Wong MC, Ding H, Wang J, Chan PS, Huang J. Prevalence and risk factors of colorectal cancer in Asia. Intest Res 2019;17:317329.

2. Hong SN. Genetic and epigenetic alterations of colorectal cancer. Intest Res 2018;16:327-337.

3. Yoon JY, Cha JM, Jeen YT; Medical Policy Committee of Korean Association for the Study of Intestinal Diseases (KASID); Quality Improvement Committee of Korean Society of Gastrointestinal Endoscopy (KSGE). Quality is the key for emerging issues of population-based colonoscopy screening. Intest Res 2018;16:48-54.

4. Rex DK, Boland CR, Dominitz JA, et al. Colorectal cancer screening: recommendations for physicians and patients from the U.S Multi-Society Task Force on Colorectal Cancer. Am J Gastroenterol 2017;112:1016-1030.

5. Robertson DJ, Lee JK, Boland CR, et al. Recommendations on fecal immunochemical testing to screen for colorectal neoplasia: a consensus statement by the US Multi-Society Task Force on Colorectal Cancer. Am J Gastroenterol 2017;112:37-53.

6. Suh M, Song S, Cho HN, et al. Trends in participation rates for the National Cancer Screening Program in Korea, 2002-2012. Cancer Res Treat 2017;49:798-806.

7. Hong S, Shin HY, Lee B, et al. Increase in the colorectal cancer screening rate by a round-mailed fecal immunochemical testing kit and associated factors in underserved regions of Korea: a community-based intervention study. Gut Liver 2020;14:323-330.

8. Jager M, Demb J, Asghar A, et al. Mailed outreach is superior to usual care alone for colorectal cancer screening in the USA: a systematic review and meta-analysis. Dig Dis Sci 2019;64:24892496.

9. Davis MM, Freeman M, Shannon J, et al. A systematic review of clinic and community intervention to increase fecal testing for colorectal cancer in rural and low-income populations in the United States-How, what and when? BMC Cancer 2018;18:40.

10. Hong NS, Kam S. Effectiveness of interventions to increase screening for gastric and colorectal cancer in Korea. Asian Pac J Cancer Prev 2014;15:9147-9151. 